\title{
Multi-Ensayos
}

Vol. 4, $\mathrm{N}^{\circ} 8$

ISSN: 2412-3285

https://multiensayos.unan.edu.ni

DOI: https://doi.org/10.5377/multiensayos.v4i8.9450

\section{La enseñanza de la innovación para el desarrollo sostenible}

\section{Teaching innovation for the sustainable development}

Oscar Antonio Chacón ${ }^{1}$

Recibido: 6 de julio de 2018, Aceptado: 25 de febrero de 2019

\section{RESUMEN}

Partiendo de la teoría planteada por autores distinguidos sobre el tema de innovación, el presente ensayo tiene como propósito de enfatizar la importancia de promover desde la educación la enseñanza de la innovación para el desarrollo sostenible. Se comparte la experiencia de la innovación promovida desde Nicaragua, a través de las universidades integrantes en el Consejo Nacional de Universidades (CNU), y más concretamente de la Bluefields Indian And Caribbean University (BICU). La BICU es una universidad comunitaria, ubicada en la Costa Atlántica de Nicaragua, y se distingue por su cosmovisión, interculturalidad, multiculturalidad y pluriculturalidad. En la BICU los estudiantes, egresados y graduados tienen la oportunidad de aprovechar para generar pequeños, medianos y grandes negocios, dinamizando el mercado local y nacional. Se comparte la experiencia de emprendimiento que se ha venido impulsando con estudiantes de la carrera de Administración de Empresas, Contaduría Pública y Finanzas y Administración de Puertos y Aeropuertos, en una relación teoría-práctica en las asignaturas de visión empresarial, y formulación y evaluación de proyectos.

Palabras claves: enseñanza; innovación; desarrollo.

\section{ABSTRACT}

Based on the theory stated by distinguished authors about innovation, this essay main purpose is to emphasize the importance of promoting from the education the learning of innovation for sustainable development. In addition, the experience of innovation promoted in Nicaragua through the universities part of the National Council of Universities (CNU), specifically at the Bluefields Indian and Caribbean University (BICU) is demonstrated. BICU is a communal university, in the Caribbean Coast of Nicaragua, it is recognized by its Cosmo vision, interculturality, and multiculturalism. At the BICU, the experience of entrepreneurship has been promoted with students of the Bachelor of Business Administration, Public Accounting and Finance and Ports and Airports Administration, focused in a combination of theory-practice through the subjects: business vision and formulation and evaluation of Projects. At the BICU, under graduated and graduated students have the opportunity to generate small, medium and large businesses, increasing the local and national market.

Keywords: teaching; innovation; development.

1 Docente de Bluefields Indian and Caribbean University. BICU. Correo electrónico: oscarantonio_35@yahoo.es Copyright (c) 2018 Revista Multi-Ensayos. 


\section{INTRODUCCIÓN}

En su teoría de las innovaciones Schumpeterer (1978), CITADO POR SANCHEZ, C. \& Rodríguez. R. (2016) introdujo el concepto de innovación al ámbito económico en la que lo define como el establecimiento de una nueva función de producción. La economía y la sociedad cambian cuando los factores de producción se combinan de una manera novedosa. Sugiere que invenciones e innovaciones son la clave del crecimiento económico.

En tanto Castro Díaz-Balart \& Delgado Fernández (2000), definen la innovación en términos de proceso como: "el proceso de creación, desarrollo, producción, comercialización y difusión de nuevos y mejores productos, procesos y procedimientos en la sociedad".

Partiendo de la teoría planteado por autores distinguidos sobre el tema de innovación el presente ensayo tiene como propósito de enfatizar la importancia de promover desde la educación la enseñanza de la innovación para el desarrollo sostenibles.

El desarrollo local, nacional y por qué no pensar en un mercado a nivel internacional como es el mercado centro americano y latino americano depende en gran manera del sistema educativo de la nación, los modelos curriculares, planes de estudios, programas deben estar ligados a las necesidades de producir nuevos productos y servicios para acaparar el mercado local y extranjeros.

No obstante, las ideas de innovaciones, debe estar ligada con el sector financiero y micro financieras de la Nación Nicaragüenses, con opciones al acceso de financiamiento a las pequeñas y medianas empresas y con el acompañamiento constante de las entidades gubernamentales que ha venido promoviendo el Gobierno de Reconciliación y Unidad Nacional (GRUN) a través del Ministerio de Economía Familia, Comunitaria, Cooperativa y Asociativa (MEFCCA), con acompañamiento técnico y financiamientos para ideas de negocios. Desarrollado a través de los Gobiernos Regionales de la Costa Caribe de Nicaragua. La Bluefields Indian And Caribbean University Universidad comunitaria e intercultural ha venido desde las aulas, en el campo promoviendo el tema de emprendurismo e innovación con los estudiantes en las diferentes carreras y ha tenido participación en las competencias nacionales desarrollado por el Consejo Nacional de Universidades. En este ensayo se presentan los productos elaborados por estudiantes con el acompañamiento de los docentes.

Es evidente que para una gestión eficiente y eficaz de la ciencia y la innovación tecnológica es imprescindible adoptar un enfoque sistémico, abarcar todo el universo de escenarios en los que presenta alguna manifestación objetiva de hombre y mujeres con pensamientos progresista de contribuir en el desarrollo socio económico de una Nicaragua libre y soberana, dejando atrás intenciones empíricas para su gestión.

\section{DESARROLLO}

\section{Panorama general del sistema educativo}

Tunnermann, C.(2008), explica que el sistema educativo nicaragüense se estructura en tres subsistemas: el primero, la Educación General Básica y Media (incluye el bachillerato y la educación normal, para formar maestros de primaria), bajo la responsabilidad del Ministerio de Educación, Cultura y Deportes (MECD); el segundo lo constituye la Educación Profesional (Técnica Media, Formación Profesional y Capacitación), bajo la responsabilidad del Instituto Nacional Tecnológico (INATEC) y el tercero, la Educación Superior, cuya instancia de coordinación y consulta es el Consejo Nacional de Universidades. 
La mayoría de las universidades privadas han creado, a su vez, dos organismos de coordinación: la Federación Nicaragüense de Universidades Privadas (FENUP) y el Consejo Superior de las Universidades Privadas (COSUP). Hay varias universidades privadas que no están afiliadas a ninguna de estas dos asociaciones.

Por su parte Brunner, J. (1990), expresa que la tasa bruta de participación en la Educación Superior en América Latina englobando 18 países latinoamericanos como: Cuba, Argentina, Venezuela, Chile, Panamá, Uruguay, Bolivia, Perú, Colombia, Costa Rica, México, Paraguay, Brasil, El Salvador, Ecuador, Nicaragua, Honduras y Guatemala se ubica en los tres primeros lugares, Cuba con un $88 \%$, Argentina $65 \%$ y Venezuela $52 \%$, en el caso particular de Nicaragua alcanza un $18 \%$. Esto significa que los tres países antes mencionados mantienen hasta el 2010 una población activa inmersa en la educación superior, significando que hasta el 2018 podría presentar una tendencia de participación significativa en relación al período de estudio presentado.

Dondy y Chichini (1995), explican que las Universidades son las instituciones más antiguas de Europa, de estas 85 instituciones fueron establecidas en 1520, que todavía perduran con rasgos reconocibles y 70 son universidades que indudablemente han cambiado y en relación a las otras instituciones lo han hecho en menor medida.

Los procesos de la innovación con respecto a la utilización de las TIC en la docencia suelen partir, la mayoría de las veces de las disponibilidades y solución tecnológicas existente; sin embargo, una equilibrada visión del fenómeno deberá de llevarnos a la integración de la innovación tecnológica en el contexto de la tradición de nuestras instituciones.

Nicaragua es uno de los países, en vías de desarrollo, que ha marcado un paso agigantado en la educación en comparación a la década de los 90 y cuenta con diez centros de educación superior públicas, miembros de la Consejo Nacional de Universidades (CNU) que brindan educación superior para población más desprotegidas en todo el territorio nacional que son financiados con el presupuesto del $6 \%$, de las cuales se mencionan:

Universidades miembros del Concejo Nacional de Universidades

\begin{tabular}{|c|c|c|c|c|}
\hline $\mathbf{N}^{\circ}$ & Razón social & Significado & Rectores & Ubicación \\
\hline 1 & UNI & Universidad Nacional de Ingenieria & Ing. Néstor Gallo Zeledón & $\begin{array}{l}\text { Managua } \\
\text { Nicaragua }\end{array}$ \\
\hline 2 & UCA & Universidad Centro Americana & José Alberto Idiáquez, S.J. & $\begin{array}{l}\text { Managua } \\
\text { Nicaragua }\end{array}$ \\
\hline 3 & UNA & Universidad Nacional Agraria & $\begin{array}{l}\text { Dr. Francisco Telémaco } \\
\text { Talavera Siles }\end{array}$ & $\begin{array}{l}\text { Managua } \\
\text { Nicaragua }\end{array}$ \\
\hline 4 & UNIAV & $\begin{array}{l}\text { Universidad Internacional de } \\
\text { Antonio Valdivieso. }\end{array}$ & $\begin{array}{l}\text { Padre Carlos Irías Amaya } \\
\text { OD }\end{array}$ & $\begin{array}{l}\text { Rivas } \\
\text { Nicaragua }\end{array}$ \\
\hline 5 & UCATSE & $\begin{array}{l}\text { Universidad Católica Agropecuaria y } \\
\text { Trópico Seco. }\end{array}$ & $\begin{array}{l}\text { Sor Sandra Margarita } \\
\text { López B. }\end{array}$ & $\begin{array}{l}\text { Estelí } \\
\text { Nicaragua }\end{array}$ \\
\hline 6 & UNAN MANAGUA & $\begin{array}{l}\text { Universidad Nacional Autónoma de } \\
\text { Nicaragua. }\end{array}$ & $\begin{array}{l}\text { MSc. Ramona Rodríguez } \\
\text { Pérez }\end{array}$ & $\begin{array}{l}\text { Managua } \\
\text { Nicaragua }\end{array}$ \\
\hline 7 & UPOLI & Universidad Politécnica de Nicaragua & PhD. Lidia Ruth Zamora & $\begin{array}{l}\text { Managua } \\
\text { Nicaragua }\end{array}$ \\
\hline 8 & $\mathrm{BICU}$ & $\begin{array}{l}\text { Bluefield's Indian And Caribbean } \\
\text { University. }\end{array}$ & $\begin{array}{l}\text { MSc. Henningston Hillary } \\
\text { Omeir Taylor }\end{array}$ & $\begin{array}{l}\text { Costa Caribe } \\
\text { Nicaragua }\end{array}$ \\
\hline
\end{tabular}




\begin{tabular}{llll}
9 URACCAN & $\begin{array}{l}\text { Universidad de la Costa Caribe Nica- } \\
\text { ragüense. }\end{array}$ & $\begin{array}{l}\text { MSc. Alta Hooker } \\
\text { Blandford }\end{array}$ & $\begin{array}{l}\text { Costa Caribe } \\
\text { Nicaragua. }\end{array}$ \\
& $\begin{array}{l}\text { Universidad Nacional Autónoma de } \\
\text { Nicaragua }\end{array}$ & $\begin{array}{l}\text { Dr. Octavio Guevara } \\
\text { Villavicencio }\end{array}$ & $\begin{array}{l}\text { León } \\
\text { Nicaragua }\end{array}$ \\
\hline
\end{tabular}

Fuente: Cuadro diseño propio en base a la información facilitada por el Consejo Nacional de Universidades en: http://www.cnu.edu.ni/universidades-miembros-del-cnu/universidades-legalmente-establecidas/

Las universidades públicas de Nicaragua que integran el Consejo Nacional de Universidades (CNU) son subvencionadas con fondos públicos, proveniente del estado, por el beneficio constitucional del $6 \%$. En estas instituciones educativas acceden el mayor número de la población estudiantil nicaragüense, y que son provienen de las diferentes regiones del pacifico y de la costa caribe.

En las universidades públicas, el tema de innovación es incipiente, ni en los modelos curriculares ni en los programas del perfil de las carreras se visibiliza la innovación como un tema específico y de alta prioridad para los estudiantes y para la Institución. Esto limita empoderar de la capacidad y habilidad intelectual los estudiantes para desarrollar productos innovadores.

\section{Revisión teórica de innovación, emprendimiento y talento humano}

Mulet, J. (2005), considera la innovación a todo "cambio que genera valor", pero es una definición demasiado general, que conviene delimitar una forma de hacerlo, es decir, que es una innovación, todo cambio basado en conocimiento que genera valor para la empresa. Pero todavía es mucho más preciso dar este nombre al resultado de un proceso complejo (lo que se verá a lo largo de toda la exposición) que lleva nuevas ideas al mercado en forma de productos o servicios y de sus procesos de producción o provisión, que son nuevos o significativamente mejorados.

Por su parte Suárez, O. M. (2004), ideó la teoría de la innovación, como el medio capaz de explicar el desarrollo económico y al analizar el cambio tecnológico diferencia entre invención e innovación. El primero se refiere a la creación de nuevos inventos, en tanto, el segundo supone la aplicación de la invención a nuevos productos o procesos de producción y su aplicación a usos comerciales o industriales. Continúa expresando que la innovación son cambios acompañados de incertidumbre y que es producido por: 1) La introducción de un nuevo bien; 2) La introducción de un nuevo método de producción; y 3) La apertura de un nuevo mercado.

Estos tres elementos conllevan a que las empresas constantemente estén ideando nuevos procesos, productos o servicio para diversificar su mercado, en correspondencia a exigencia de los consumidores de bienes y servicios.

En su teoría de las innovaciones Schumpeterer (1978), Citado por Sanchez, C. Rodríguez R.(2017) introdujo el concepto de innovación al ámbito económico en la que lo define como el establecimiento de una nueva función de producción. La economía y la sociedad cambian cuando los factores de producción se combinan de una manera novedosa. Sugiere que invenciones e innovaciones son la clave del crecimiento económico.

En tanto Castro Díaz-Balart \& Delgado Fernández (2000), Citado por Sanchez, C. Rodríguez R. (2017) definen la innovación en términos de proceso como: "el proceso de creación, desarrollo, producción, comercialización y difusión de nuevos y mejores productos, procesos y procedimientos en la sociedad". Para Rodríguez, R. (2011), emprender es un término que tiene múltiples acepciones, según el contexto en que se le emplee será la connotación que se le adjudique. En el ámbito de los negocios el emprendedor 
es un empresario, es el propietario de una empresa comercial con fines de lucro; Finley (1990) lo describe como alguien que se aventura en una nueva actividad de negocios; Say (1800) - citado por Drcker (1989) - lo clasifica como un "buen administrador".

Plantea Drucker, P. F. (2001) que, para innovar en un negocio determinado, las relaciones y la estructura deben ser diseñadas para que se concentren y se fomenten las actitudes emprendedoras. Los incentivos, compensación y toma de decisiones sobre el personal deben estar orientados a promover el espíritu emprendedor. Las empresas no deben mezclar las unidades gerenciales con las emprendedoras, ni hacer de la innovación un objetivo para quienes se encargan de temas de control de la compañía. Tampoco deberían "comprar" su clima emprendedor, adquiriendo otras empresas. Una nueva empresa debe tomar en cuenta los requerimientos fundamentales de gerencia: Enfocarse en el mercado, planificación financiera, que anticipe los requerimientos de flujo de caja y necesidades de capital. Un equipo gerencial de primera, una decisión, por parte del emprendedor fundador, acerca de su rol y sus relaciones en la empresa.

Para Becerra Rodríguez, F., \& Álvarez Giraldo, C. M. (2011), la innovación le permite a una organización crear valor a través del desarrollo de un nuevo conocimiento o por usos nuevos del conocimiento existente, mediante un proceso problema o necesidad que debe ser atendida, la concepción de la idea que dará la solución, su adopción, e importancia del recurso humano en el éxito de las empresas es ampliamente reconocida en la literatura (Fabi, Raymond y Lacoursière, 2007; Hewitt, 2006; Laursen y Foss, 2003; Michie y Sheehan, 1999; Vinding, 2006). Aspectos como las capacidades y competencias distintivas de las personas que laboran en la organización resultan cruciales para la competitividad empresarial, aspecto que ha sido subrayado desde el surgimiento de la teoría de recursos y capacidades (Barney, 1991).

Según Chiavenato, I., \& Villamizar, G. (2002), que consta de 12 aspectos esenciales de la gestión del talento humano, los cuales se describen:

1. Nueva filosofía de acción.

2. Nítida y rápida tendencia al downsizing.

3. Transferencia de un área de servicio en un área de consultoría interna.

4. Transferencia gradual de decisiones y acciones de la ARH hacia la gerencia de líneas.

5. Intensa conexión con el negocio de la empresa.

6. Énfasis en la cultura participativa y democrática en las organizaciones

7. Utilización de mecanismos de motivación y de realización personal.

8. Adecuación de las prácticas y políticas de RH a las diferencias individuales de las personas.

9. Viraje completo en dirección a/ cliente, sea interno o externo.

10. Gran preocupación por /a creación de valor en la empresa.

11. Preocupación por preparar la empresa y a las personas para e/ futuro

12. Utilización intensiva del benchmarking como estrategia de mejoramiento continuo de los procesos y servicios. 


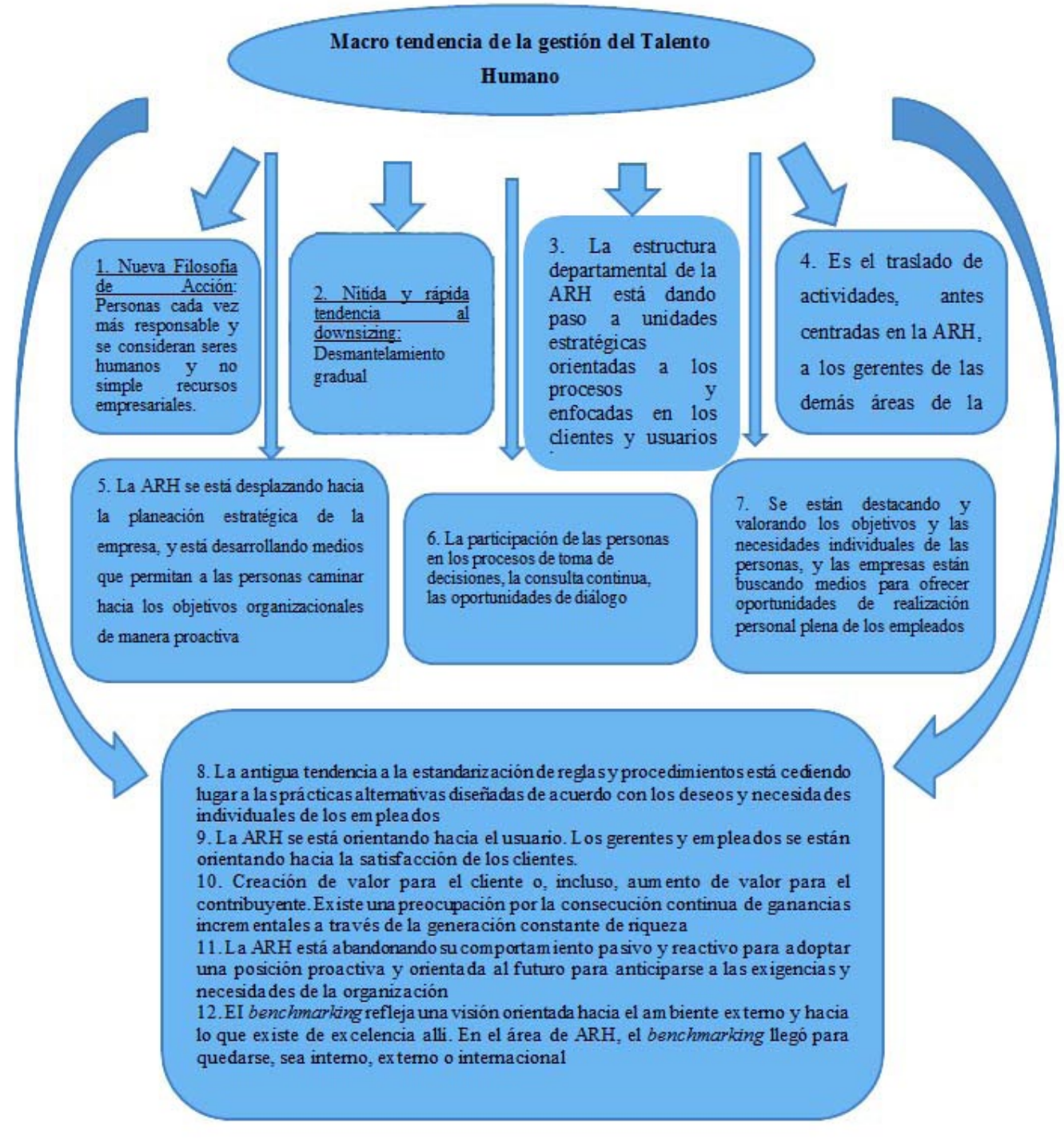

Fuente: Figura diseño propio

Fredy, R \& Claudia A. (2011), expresan que el talento humano se expresa en la importancia del recurso humano en el éxito de las empresas es ampliamente reconocida en la literatura (Fabi, Raymond y Lacoursière, 2007; Hewitt, 2006; Laursen y Foss, 2003; Michie y Sheehan, 1999; Vinding, 2006). Aspectos como las capacidades y competencias distintivas de las personas que laboran en la organización resultan cruciales para la competitividad empresarial, aspecto que ha sido subrayado desde el surgimiento de la teoría de recursos y capacidades (Barney,1991).

Y la innovación permite a la organización crear valor a través del desarrollo de un nuevo conocimiento o por usos nuevos del conocimiento existente, mediante un proceso o por uso de nuevos conocimientos existentes, mediante un proceso que comprenda la definición de un problema o necesidad que debe ser atendido, la concepción de la idea que dará la solución, su adquisición e implementación y su comercialización (Claver Llopis, García y Molina; 1987. Evan y Black; 1967, Wolfe; 1994).

\section{Los procesos de innovación educativa}

Salina, J. (2004), afirma que los procesos de innovación respecto a la utilización de las TIC en la docencia universitaria suelen partir, la mayoría de las veces, de las disponibilidades y soluciones tecnológicas 
existentes. Sin embargo, una equilibrada visión del fenómeno debería llevarnos a la integración de las innovaciones tecnológicas en el contexto de la tradición de nuestras situaciones; instituciones que, no olvidemos, tienen una importante función educativa. Debemos considerar la idiosincrasia de cada una de las instituciones al integrar las TIC en los procesos de la enseñanza superior. También que la dinámica de la sociedad puede dejarnos al margen.

Como plantea Ibáñez, J. S. (2004), los procesos de innovación respecto a la utilización de las TIC en la docencia universitaria suelen partir, la mayoría de las veces, de las disponibilidades y soluciones tecnológicas existentes. Sin embargo, una equilibrada visión del fenómeno debería llevarnos a la integración de las innovaciones tecnológicas en el contexto de la tradición de nuestras instituciones.

Según Balmaseda, E, Elguezabal. (2008) en su estudio hacen referencia que existe una serie de modelos de la innovación y sobre estos varios autores han tratado de clasificarlos. En la Tabla 2 se describen los aportes de cada uno de los autores que han planteado sus opiniones al respecto.

Tabla 2. Clasificación y modelos ofrecidos por distintos autores sobre el proceso de innovación

\begin{tabular}{|c|c|c|c|}
\hline $\mathbf{N}^{\circ}$ & Autores & $\mathbf{N}^{\circ}$ & Clasificación de Modelo del Proceso de \\
\hline \multirow{5}{*}{1} & \multirow{5}{*}{ Saren,M.A.(1993) } & 1 & Modelo de etapas departamentales (Departamental Stage \\
\hline & & 2 & Modelos de etapas de actividades (Activity Stage Models) \\
\hline & & 3 & Modelo de etapa de decisión (Desition Stage Models) \\
\hline & & 4 & Modelo de etapa de Conversión (Convertion Process) \\
\hline & & 5 & Modelo de Repuestas (Response Models) \\
\hline \multirow{4}{*}{2} & \multirow{4}{*}{ Fornest,J(1991) } & 1 & Modelo de etapas (Stage Models) \\
\hline & & 2 & $\begin{array}{l}\text { Modelo de conversión y modelo de empuje de la tecnología /Tiron } \\
\text { de la demanda (Conversion Models Ans Tecnology - Push) }\end{array}$ \\
\hline & & 3 & Modelo integradores (Integrative Models). \\
\hline & & 4 & Modelo de Decisión (Decisión Models). \\
\hline \multirow{5}{*}{3} & \multirow{5}{*}{ Rothwell,R.(1994) } & 1 & Proceso de innovación de primera generación: empuje de la \\
\hline & & 2 & Proceso de Innovación de segunda generación: Tiron de la \\
\hline & & 3 & $\begin{array}{l}\text { Proceso de Innovación de la Tercera generación: Modelo Interactivo } \\
\text { (Caupling Models) }\end{array}$ \\
\hline & & 4 & Proceso de Innovación de la Cuarta generación: Proceso de \\
\hline & & 5 & $\begin{array}{l}\text { Proceso de Innovación de Quinta generación (System } \\
\text { Integretionand Newworking) }\end{array}$ \\
\hline \multirow{3}{*}{4} & \multirow{3}{*}{$\begin{array}{l}\text { Padmore, T, Schuetze, H y } \\
\text { Gibson, H (1998) }\end{array}$} & 1 & Modelo Linea (Linear Models) \\
\hline & & 2 & Modelo de enlace en Cadena (Chain link models) \\
\hline & & 3 & Modelo en ciclo (Cycle Models) \\
\hline
\end{tabular}

\section{Innovación en la Bluefields Indian and Caribbean University BICU}

Bluefields Indian And Caribbean University (BICU), es una universidad caribeña y autóctona comunitaria de la Costa Caribe de Nicaragua, financiada el $100 \%$ con presupuesto del estado. Cuenta con presupuesto reducido en comparación a las otras universidades miembros de la CNU, esto obliga a las autoridades que gerencian las actividades académicas a priorizar entre las prioridades las actividades más importantes, limitando en rubros que no dejan de ser importantes como es el tema de la innovación. 
La BICU en su presupuesto no cuenta con una partida presupuestaria para implementar la innovación desde las aulas de clases con el involucramiento de los estudiantes y que esto contribuya en el desarrollo profesional de los egresados y de los estudiantes en su proceso de formación, crear en ellos una visión de empresarios, no buscar empleo sino crear fuentes de empleos a través de sus propias empresas sin distingo de su tamaño o capital social de constitución. De esta forma las universidades estarían contribuyendo al desarrollo económico del país a través de las MIPYMES. Siendo los principales protagonistas los graduados y graduadas.

No obstante, los docentes de la Facultad de Ciencias Económicas y Administrativas, han venido impulsando el tema innovación en la asignatura de visión empresarial y formulación y evaluación de proyectos, en teoría y práctica en la carrera de Administración de Empresas. Sin embargo, esto únicamente se limita a la elaboración de productos, sin culminar el proceso de la pantentizacion de los productos para comercializarlo con la debida autorización del Ministerio de Salud (MINSA) y las instancias correspondientes.

A continuación, se detalla el listado de productos elaborados por docentes y estudiantes innovadores de la Facultad de Ciencias Económicas y la Escuela de Ciencias de la Educación

1. Vino de Nancite.

2. Vino de Toronja.

3. Crema Painkira para cara elaborado con miskitu batana.

4. Crema rejuvenecedora de badu.

5. Alimentos para Aves.

6. Crema Miskitu batana para la piel.

7. Dibujos y Maquetas.

Los productos fueron elaborados bajo la supervisión y apoyo de la profesora Isabel Guadamuz Pérez, con más de 20 semestres de experiencias en la docencia, mujer emprendedora y empresaria que ha venido contribuyendo con sus conocimientos en el aula de clase. Estos productos han sido expuestos en jornadas universitarias, ferias territoriales desarrolladas con el Ministerio de Educación y en la jornada de investigación científica promovidas por la CNU, en la Ciudad de Managua, en el 2016 y 2017. Así mismo, los docentes Carolina Cárdenas Guido y Jorge Frank Pérez, de Ciencias de la Educación.

Beatty, E. N., \& Escofet, L. O. (1996), en la invención e innovación hay muchos factores sociales e institucionales que afectan la propensión de los individuos a inventar y la capacidad de las sociedades de innovar. De estos factores, uno de los más tangibles es el sistema de patentes, que en esencia, ofrecen al inventor derechos temporales de monopolio, gracias a los cuales tiene poder exclusivo de producir o utilizar el procedimiento patentado. Los sistemas de patentes surgen de la naturaleza del conocimiento como bien público. La teoría económica sostiene que los mercados competitivos no reembolsarán adecuadamente a los productores de conocimiento nuevo los costos implicados en la creación.

Plantea García, A (2008), que la propiedad intelectual en las universidades, siempre está relacionado con dos aspectos importantes: Investigación y Extensión, por cuanto la propiedad intelectual busca proteger la creatividad o la innovación, el esfuerzo y la inversión, así como el conocimiento de la exclusividad, la utilidad económica y social.

Muchos factores sociales e institucionales afectan la propensión de los individuos a inventar y la capacidad de las sociedades de innovar. De estos factores, uno de los más tangibles es el sistema de 
patentes, que en esencia, ofrece al inventor derechos temporales de monopolio, gracias a los cuales tiene poder exclusivo de producir o utilizar el procedimiento patentado. Los sistemas de patentes surgen de la naturaleza del conocimiento como bien público. La teoría económica sostiene que los mercados competitivos no reembolsarán adecuadamente a los productores de conocimiento nuevo los costos implicados en la creación.

\section{CONCLUSIÓN}

El tema desarrollado en el presente ensayo demuestra la importancia de generar conocimientos a través de investigación, desde un nivel básico de la educación superior de grado, y cómo estos conocimientos son concretizados en la solución de un problema que atañe a la región del Caribe Norte y Sur de Nicaragua. En ambas regiones abundan múltiples problemas en el sector de la economía regional, esto recae serios compromisos y la vez oportunidades a las universidades miembros del CNU, de la RAACN Y RAAS, impulsar investigaciones que contribuyan en minimizar los problemas que son objeto de investigación y que los recursos financieros de investigaciones del $6 \%$ estén dirigidos para estos fines primordiales.

Los procesos de enseñanza y aprendizaje que ha venido implementando las universidades de la educación superior, subsidiado con el presupuesto de la república de Nicaragua, se ha venido dinamizando con la aplicación de nuevas tecnologías y metodologías pedagógicas modernas, lo que se ha visto demostrado en los estudiantes a través de la prácticas con la elaboración de productos innovadores, como una forma de incentivación, de generar fuentes de ingresos, nuestro Gobierno a través del CNU ha venido desarrollando competencia a nivel nacional como internacional, a través de ferias educativas en representación de nuestro país en donde BICU ha tenido participación.

Estos impulsos gigantesco en la educación superior, que inclusive se ha venido promoviendo en los últimos 10 años en la educación primaria y secundaria el Gobierno de Reconciliación y Unidad Nacional ha permitido a nuestros profesionales egresado de BICU "Bluefields Indian And Caribbean University" se gradúen con visión de emprendedores e innovadores de nuevos productos y servicios para atender a la población de la Costa Caribe y del territorio nacional y una muestra visible y tangible, que los estudiantes de BICU en las diferentes carreras y en especial en la Facultad de Ciencias Económicas y Administrativas, han ensayado la elaboración de diferentes tipos de productos. Hoy en día la Facultad económica cuenta con estudiantes emprendedores e innovadores en ideas de negocios que están en el mercado prestando servicios y productos con negocios propios, contribuyendo con la nación nicaragüense en la generación de fuentes de empleos directos e indirectos y la generación de impuesto tributarios a través de sus obligaciones y retenciones en su calidad de empleadores en cumplimiento a la ley 822 ley de concertación tributaria y su reglamento.

\section{BIBLIOGRAFÍAS CONSULTADAS}

Balmaseda, E, y Elguezabal (2008) ). Evolución de las propuestas sobre el proceso de innovación: ¿ Qué se puede concluir de su estudio? Investigaciones Europeas de dirección y economía de la empresa, 14(2), 127-138

Beatty, E. N., \& Escofet, L. O. (1996). Invención e innovación: ley de patentes y tecnología en el México del siglo XIX. Historia mexicana, Vol. 45, No. 3 (Jan. - Mar., 567-619. Published by: El Colegio de Mexico

Becerra Rodríguez, F., \& Álvarez Giraldo, C. M. (2011). EL TALENTO HUMANO Y LA INNOVACIÓN EMPRESARIAL EN EL CONTEXTO DE LAS REDES EMPRESARIALES: EL CLÚSTER DE PRENDAS DE VESTIR EN CALDAS-COLOMBIA. Estudios Gerenciales, 27(119), 209-234. https://doi.org/10.1016/ S0123-5923(11)70164-4 
Brunner, J. (1,990), La educación superior en américa latina.C., T. B. (1193). Perspectiva del desarrollo de la educación superior

Chiavenato, I., \& Villamizar, G. (2002). GESTION DEL TALENTO HUMANO

Dondy y Chichini $(1,995)$ La educación superior en Nicaragua. Nicaragua: www.cnu.udu.ni.

Drucker, P. F. (2001) O esencial de Drukerl Lisboa: Actual Editora

Fornest,J (1991) Clasificación de modelos de la innovación

Fredy, R , Claudia A.( 2011) El Talento Humano y la Innovación Empresarial en el contexto de las redes empresariales, clauster de prendas de prendas de vestir en caldas Colombia .

García, A (2008) economía universitaria. Facultad de Derecho y Ciencias Políticas, 38(108).

Ibáñez, J. S. (2004), Educación Superior y Tecnología digital: Consideraciones y reflexiones. In Nueva tecnologías y educación.

Mulet, J. (2005) La innovación, concepto e importancia económica. In VI Congreso de Economía de Navarra.

Padmore, T, Schuetze,.H y Gibson (1998) Clasificación de modelos y procesos sobre la innovación.

Rodríguez, R. (2011). El emprendedor de éxito (Cuarta ed.). Monterrey, México: Mc Graw-Hill. Obtenido de https://periodicooficial.jalisco.gob.mx/sites/periodicooficial.jalisco.gob.mx/files/ el_emprendedor_de_exito-_rafael_alcaraz_rodriguez.pdf

Rothwell,R.(1994) Clasificación de los procesos sobre la innovación.

Salina, J. (2004), innovación docente y el uso de las TIC en la enseñanza universitaria RUSC. Univerities and Knowledge Society Journal,. (1-1).

Saren,M.A.(1993, Clasificación de modelos y procesos sobre la innovación.

Sanchez,C.Rodríguez R.(2017) La Innovación Revista Cienfuego Universidad y Sociedad, Habana Cuba Editorial ,106

Suárez, O. M. (2004) Shumppeter,Innovación y dererminimo ecnologico. Sicentia et teachnica.2 (25), tecnológico.

Tunnermann, C. (2008) La educación superior en Nicaragua. Avaliação: Revista da (2), 337-367.

http://www.cnu.edu.ni/universidades-miembros-del-cnu/universidades-legalmente-establecidas/

\section{ANEXOS}

\section{Maqueta edificio BICU recinto Bilwi Puerto Cabeza ubicado en el Barrio Rosario Murillo}

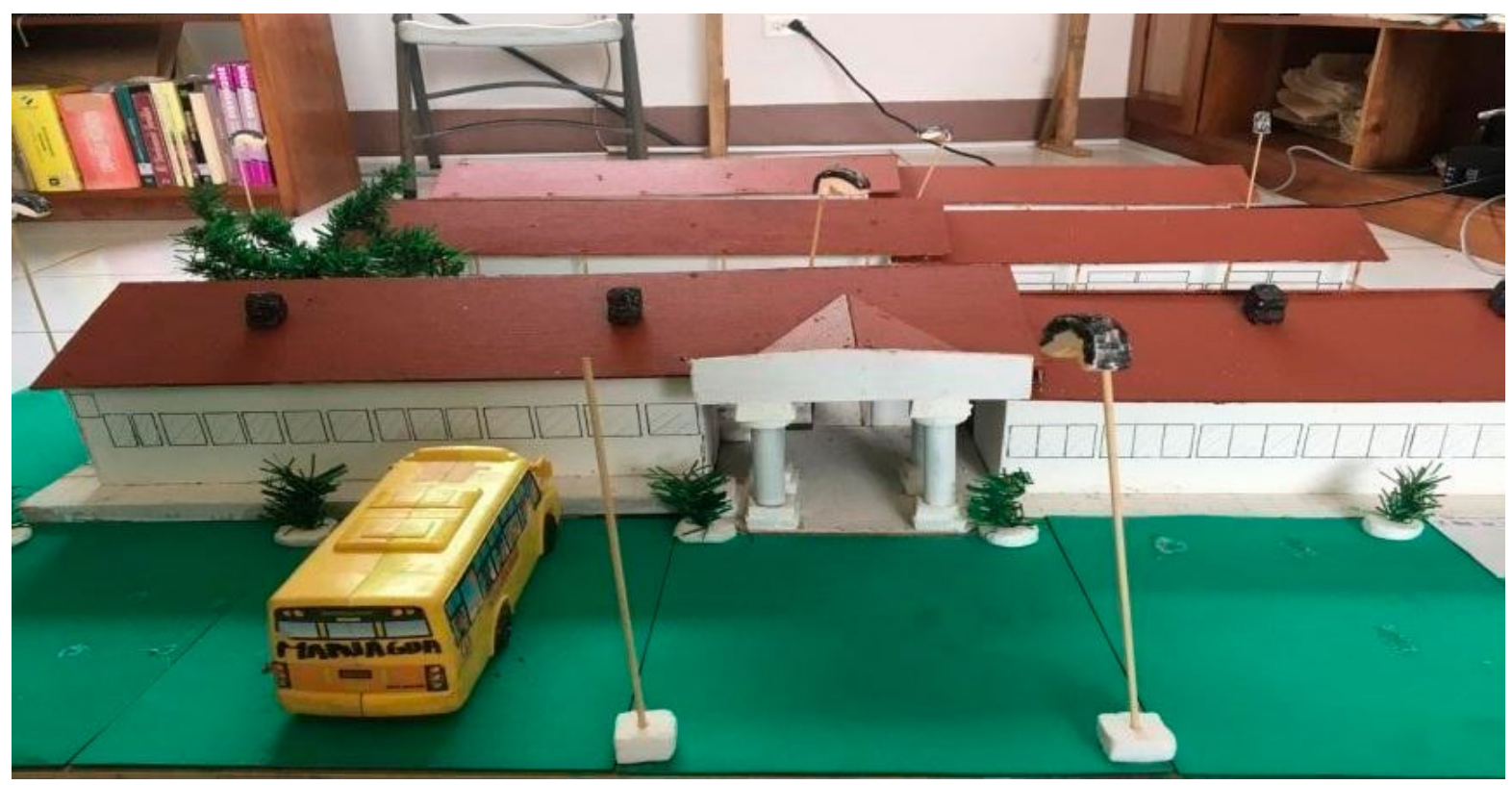

Fuente: Jarol Romel, Mario Amador, Mauricio Nicolás y Jefer Smith 
Diseño de Embarcaciones Utilizado en los Bancos de Pesca en los Cayos Mislitu en la RACCN

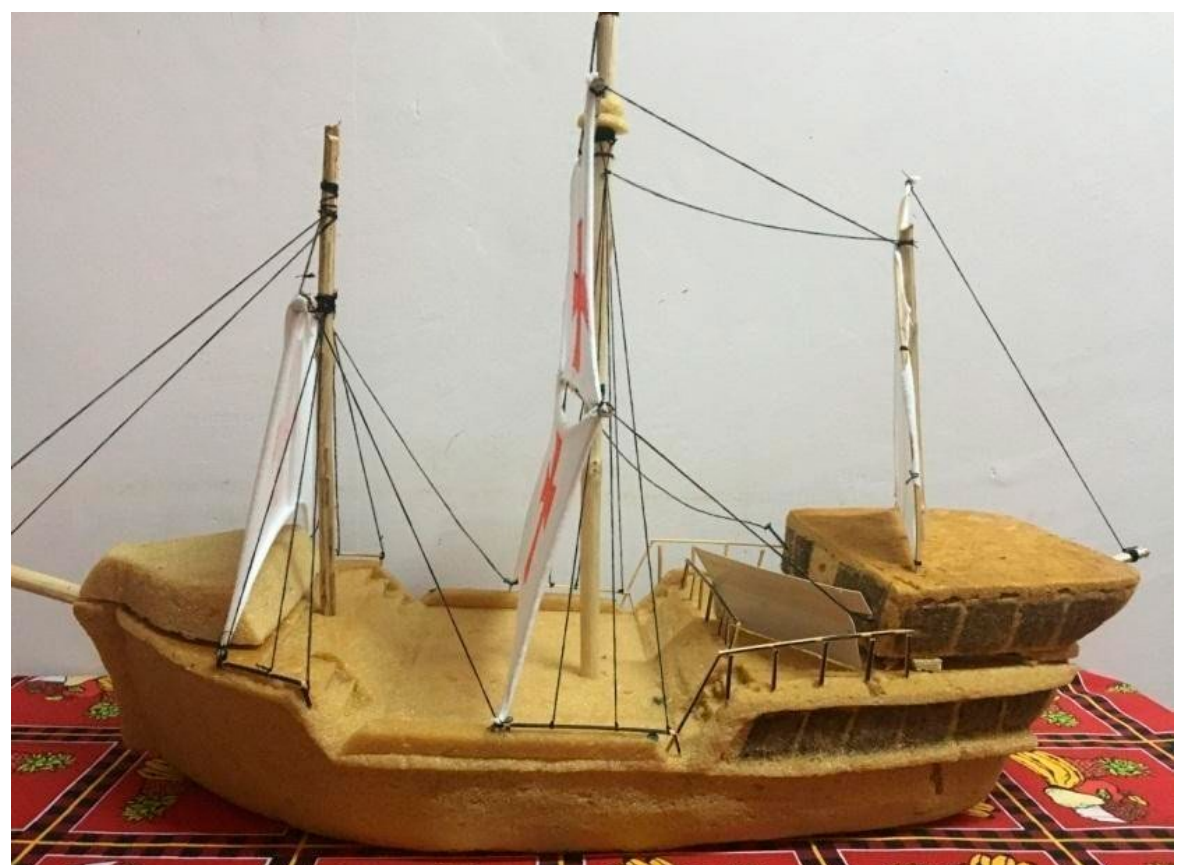

Fuente: Escuela Ciencias de la Educación BICU Bilwi.

Diseño Casa de habilitaciones Urbanas utilizadas en los municipios de la Costa Caribe Norte

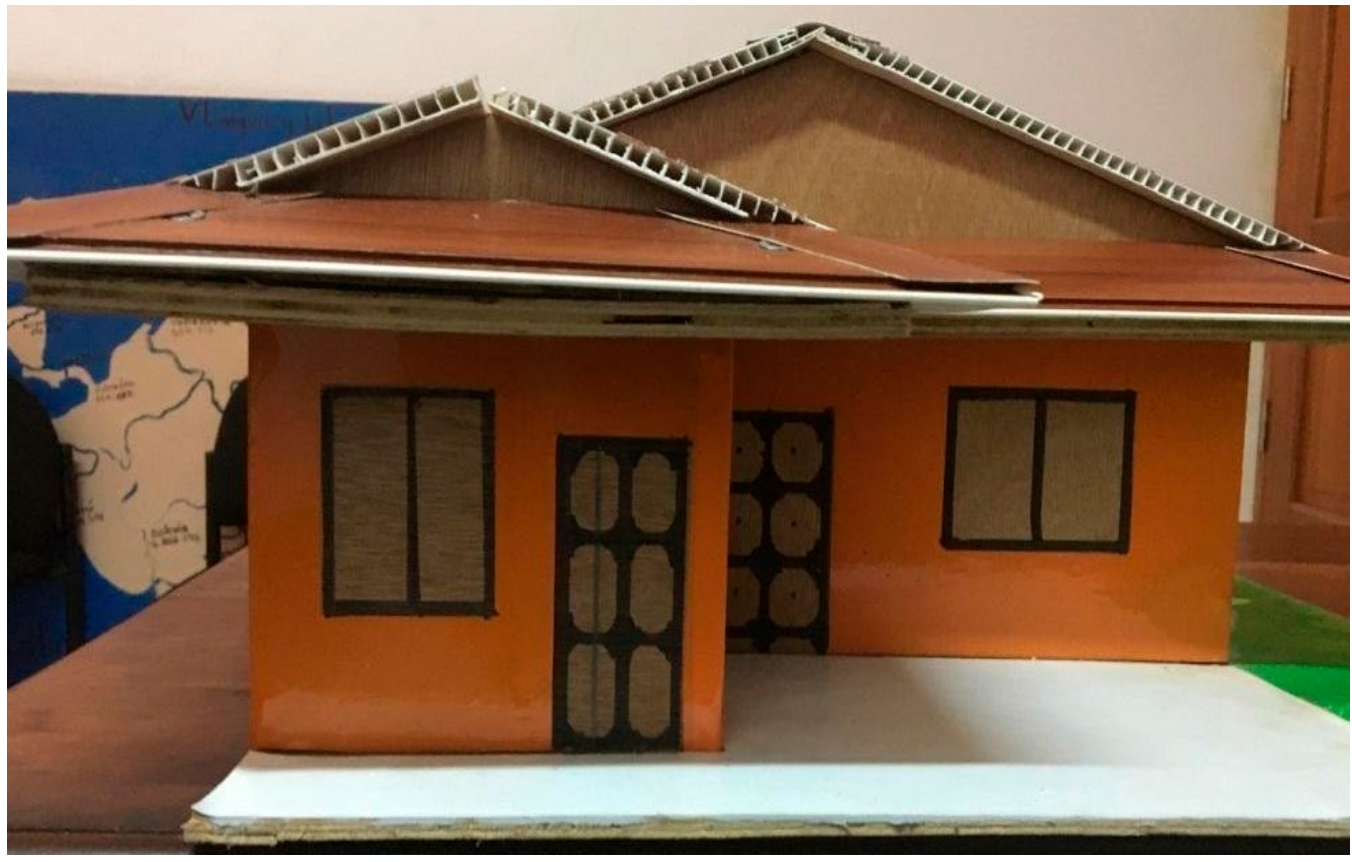

Autores: Estudiantes de Construcción Técnico Medio BICU Bilwi. 
Construcciones, y habitaciones tradicionales de los hermanos Miskitu, Sumo, Mayagna en las Comunidades indígenas de la Región Norte

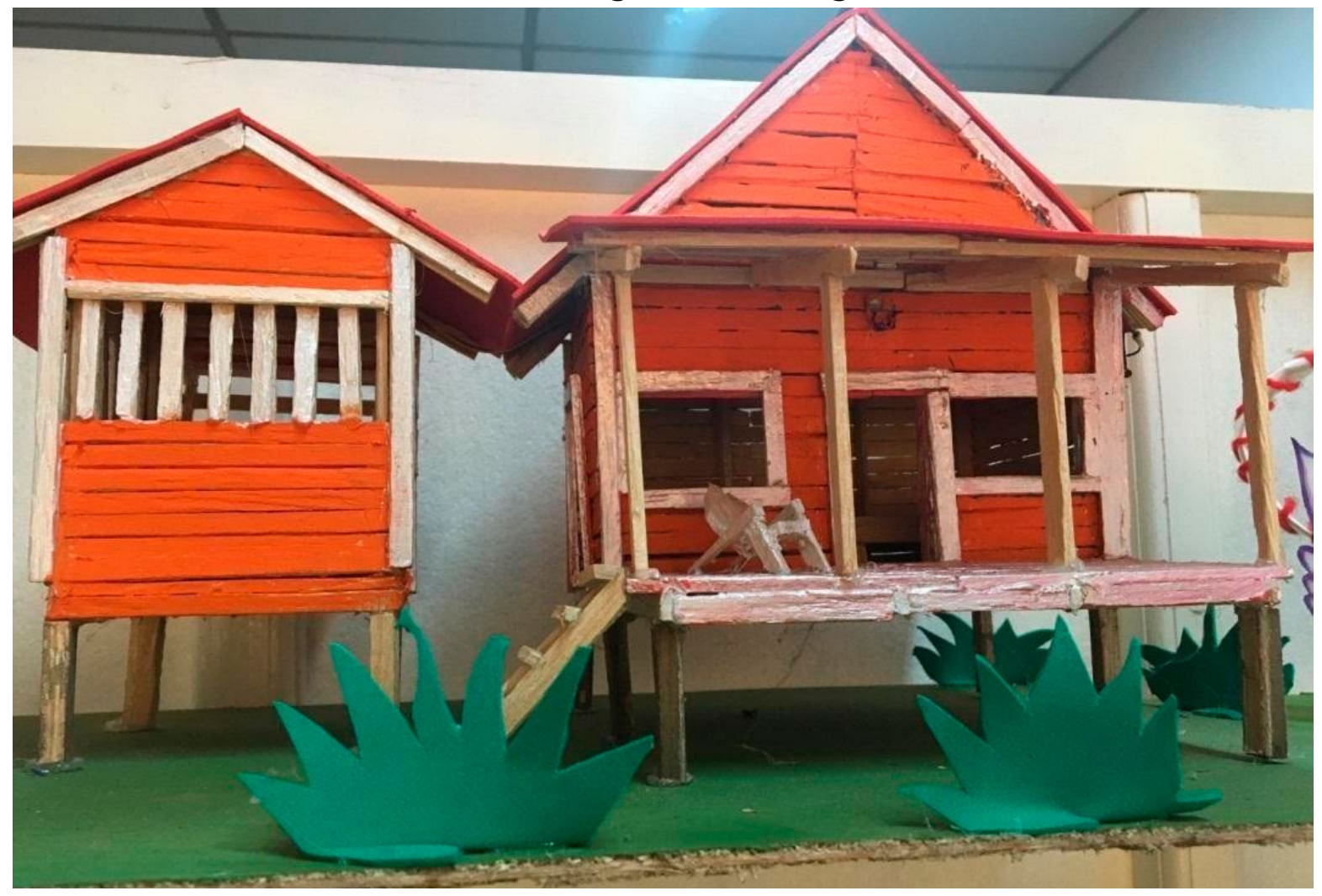

